\title{
Performance Analysis of Neural Network Architecture Combined with DWT for Image Compression
}

\author{
Murali Mohan.S \\ Associate Professor \\ Dept. of ECE,SVCET \\ Chittoor,A.P.,INDIA
}

\author{
P.Satyanarayana, PhD. \\ Professor \\ College of Engineering \\ S.V.University, Tirupati
}

\begin{abstract}
Neural networks are significantly used in signal and image processing techniques for pattern recognition and template matching. In this work neural networks are used for image compression. In order to improve the performances image compression algorithm, DWT is combined with NN for achieving better MSE and increase in compression ration greater than $100 \%$. NN architecture achieves maximum of $98 \%$ with use of four neurons in the hidden layer, with selection of LL sub band only the compression is improved by another $75 \%$. The proposed architecture is analyzed for 20 images and MSE is found to be improved by a factor of $20 \%$. Daubechies wavelet filter and Haar wavelet filters are used for DWT, input layer with one hidden layer and output layer consisting of tansig and purelin function us used for compression. The design proposed is suitable for high resolution image compression.
\end{abstract}

\section{General Terms}

Wavelet Transforms, Image processing, MSE

\section{Keywords}

DWT, neural network, image compression, hybrid technique.

\section{INTRODUCTION}

Image compression is one of the most promising subjects in image processing. Images captured need to be stored or transmitted over long distances. Raw image occupies memory and hence need to be compressed. With the demand for high quality video on mobile platforms there is a need to compress raw images and reproduce the images without any degradation. Several standards such as JPEG200, MPEG-2/4 recommend use of Discrete Wavelet Transforms (DWT) for image transformation [1] which leads to compression with when encoded. Wavelets are a mathematical tool for hierarchically decomposing functions in multiple hierarchical sub bands with time scale resolutions. Image compression using Wavelet Transforms is a powerful method that is preferred by scientists to get the compressed images at higher compression ratios with higher PSNR values [2]. It is a popular transform used for some of the image compression standards in lossy compression methods. Unlike the discrete cosine transform, the wavelet transform is not Fourier-based and therefore wavelets do a better job of handling discontinuities in data.

On the other hand, Artificial Neural Networks (ANN) for image compression applications has marginally increased in recent years. Neural networks are inherent adaptive systems [3][4][5][6]; they are suitable for handling nonstationaries in image data. Artificial neural network can be employed with success to image compression. Image Compression Using Neural Networks by Ivan Vilovic [7] reveals a direct solution method for image compression using the neural networks. An experience of using multilayer perceptron for image compression is also presented. The multilayer perceptron is used for transform coding of the image. Image compression with neural networks by J. Jiang [8] presents an extensive survey on the development of neural networks for image compression which covers three categories: direct image compression by neural networks; neural network implementation of existing techniques, and neural network based technology which provide improvement over traditional algorithms. Neural Networks-based Image Compression System by H. Nait Charif and Fathi. M. Salam [9] describes a practical and effective image compression system based on multilayer neural networks. The system consists of two multilayer neural networks that compress the image in two stages. The algorithms and architectures reported in these papers sub divided the images into sub blocks and the sub blocks are reorganized for processing. Reordering of sub blocks leads to blocking artifacts. Hence it is required to avoid reorganization of sub blocks. One of the methods was to combine neural networks with wavelets for image compression. Image compression using wavelet transform and a neural network was suggested previously [10]. Wavelet networks (WNs) were introduced by Zhang and Benveniste [11], [12] in 1992 as a combination of artificial neural networks and wavelet decomposition. Since then, however, WNs have received only little attention. In the wavelet networks, the basis radial functions in some RBF-networks are replaced by wavelets. Szu et al. [13], [14] have shown usage of WNs for signals representation and classification. They have explained how a set of WN, "a super wavelet", can be produced and the original ideas presented can be used for the assortment of model. Besides, they have mentioned the big compression of data achieved by such a representation of WN's. Zhang [15] has proved that the WN's can manipulate the non-linear regression of the moderately big dimension of entry with the data of training. Ramanaiah and Cyril [16] in their paper have reported the use of neural networks and wavelets for image compression. In their work, the image is decomposed using DWT into four sub bands, and the neural network compresses the individual sub band and hence blocking artifacts error is minimized in the reconstructed image. Image decomposition using DWT into multiple sub bands leads to delay in compression, as the decomposition of image leads to multiple hierarchical sub blocks. In this paper novel approach for image compression using wavelets and neural networks is proposed. The input image is decomposed into four sub bands of LL, LH, HL and HH. 
Only the LL sub band is further decomposed in hierarchical sub bands until the sub band size is $8 \times 8$. The sub bands after decomposition using DWT are chosen based on information content and is further compressed using multilayered neural network architecture, thus minimizing the delay in compression.

Section II presents theoretical background on neural networks and DWT. Section III discusses the proposed image compression technique, section IV presents the implementation details and section $\mathrm{V}$ discuss the results and conclusion is presented in section VI.

\section{Neural networks and DWT}

In this section, neural network architecture for image compression is discussed. Feed forward neural network architecture and back propagation algorithm for training is presented. DWT based image transformation and compression is also presented in this section. Compression is one of the major subject of research, the need compression is discussed as follows: Uncompressed vi of size $640 \times 480$ resolution, with each pixel of $8 \mathrm{bi}$ bytes), with 24 fps occupies 307.2 Kbytes per im (frame) or 7.37 Mbytes per second or 442 Mbytes minute or 26.5 Gbytes per hour. If the frame rate increased from $24 \mathrm{fps}$ to $30 \mathrm{fps}$, then for $640 \mathrm{x}$ resolution, 24 bit ( 3 bytes) colour, 30 fps occupies 92 Kbytes per image (frame) or 27.6 Mbytes per seconc 1.66 Gbytes per minute or 99.5 Gbytes per hour. Give 100 Gigabyte disk can store about 1-4 hours of $\mathrm{r}$ quality video, With channel data rate of $64 \mathrm{Kbits} / \mathrm{sec}-4$ $438 \mathrm{secs} / \mathrm{per}$ frame transmission. For HDTV with 72 1280 pixels/frame, progressive scanning at 60 frame $1.3 \mathrm{~Gb} / \mathrm{s}$ - with $20 \mathrm{Mb} / \mathrm{s}$ available $-70 \%$ compress required $-0.35 \mathrm{bpp}$. In this work a novel architecture ba on neural network and DWT is proposed.

\subsection{Feed forward neural network architecture for image compression}

An Artificial Neural Network (ANN) is an informationprocessing paradigm that is inspired by the way biological nervous systems, such as the Brian, process information [16]. The key element of this paradigm is the novel structure of the information processing system. The basic architecture for image compression using neural network is shown in figure 1 . The network has input layer, hidden layer and output layer. Inputs from the image are fed into the network, which are passed through the multi layered neural network. The input to the network is the original image and the output obtained is the reconstructed image. The output obtained at the hidden layer is the compressed image. The network is used for image compression by breaking it in two parts as shown in the Figure 1. The transmitter encodes and then transmits the output of the hidden layer (only 16 values as compared to the 64 values of the original image). The receiver receives and decodes the 16 hidden outputs and generates the 64 outputs. Since the network is implementing an identity map, the output at the receiver is an exact reconstruction of the original image.

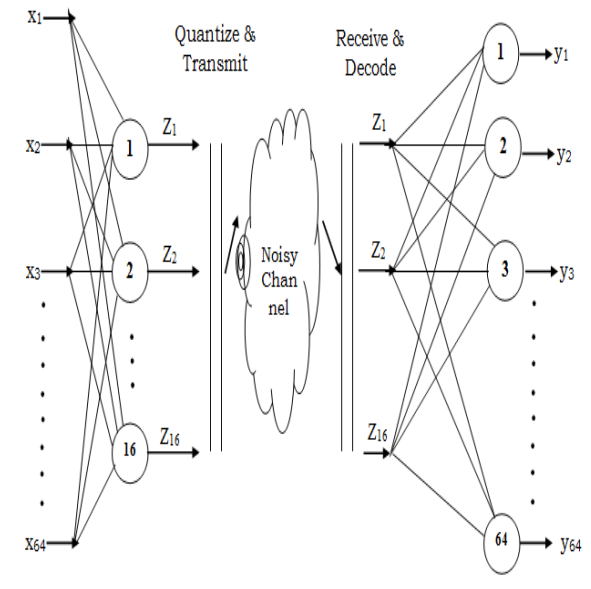

Figure 1 Feed forward multilayered neural network architecture [17]

Three layers, one input layer, one output layer and one hidden layer, are designed. The input layer and output layer are fully connected to the hidden layer. Compression is achieved by designing the network such that the number of neurons at the hidden layer is less than that of neurons at both input and the output layers. The input image is split up into blocks or vectors of 8 X8, 4 X 4 or 16 X 16 pixels. Back-propagation is one of the neural networks which are directly applied to image compression coding [18][19][20]. In the previous sections theory on the basic structure of the neuron was considered. The essence of the neural networks lies in the way the weights are updated. The updating of the weights is through a definite algorithm. In this paper Back Propagation (BP) algorithm is studied and implemented. The algorithm is applied for the supervised learning that is a desired output will be applied to Neural Architecture. The target is represented as $d_{i}$ (desired output) for the $i^{\text {th }}$ output unit. The actual output of the layer is given by $a_{i}$. Thus the error or cost function is given by [21]

$$
E=\frac{1}{2}\left(a_{i}^{2}-d_{i}\right)^{2}
$$

This process of computing the error is called a forward pass. How the output unit affects the error in the ith layer is given by differentiating equation 2.5 by $a_{i}$

$$
\frac{\partial E}{\partial a_{i}}=\left(a_{i}^{2}-d_{i}\right)
$$

The equation 2.6 can be written in the other form as

$\partial_{i}=\left(a_{i}^{2}-d_{i}\right) d\left(a_{i}^{2}\right)$

where $\mathrm{d}\left(a_{i}\right)$ is the differentiation of the $a_{i}$. The weight update is given by

$$
\Delta w_{i j}=\eta \partial_{i} a_{i}^{1}
$$

Where $a_{i}^{l}$ is the output of the hidden layer or input to the output neuron and $\eta$ is the learning rate [1]. This error has to propagate backwards from the output to the input. The $\partial$ for the hidden layer is calculated as

$\partial_{\text {hiddenlaye }}=d\left(a_{i}^{1}\right) \sum w_{i j} \partial_{i}$

Weight update for the hidden layer with new $\partial$, will be done using equation 2.8. Equation $2.5-2.9$ depend on the 
number of the neurons present in the layer and the number of layers present in the network.

\subsection{DWT architecture for image compression}

The DWT represents the signal in dynamic sub-band decomposition. Generation of the DWT in a wavelet packet allows sub-band analysis without the constraint of dynamic decomposition. The discrete wavelet packet transform (DWPT) performs an adaptive decomposition of frequency axis. The specific decomposition will be selected according to an optimization criterion. The Discrete Wavelet Transform (DWT), based on time-scale representation, provides efficient multi-resolution subband decomposition of signals. It has become a powerful tool for signal processing and finds numerous applications in various fields such as audio compression, pattern recognition, texture discrimination, computer graphics [22][23][24] etc. Specifically the 2-D DWT and its counterpart 2-D Inverse DWT (IDWT) play a significant role in many image/video coding applications. Figure 2 shows the DWT architecture, the input image is decomposed into high pass and low pass components using HPF and LPF filters giving rise to the first level of hierarchy. The process is continued until multiple hierarchies are obtained. A1 and D1 are the approximation and detail filters.
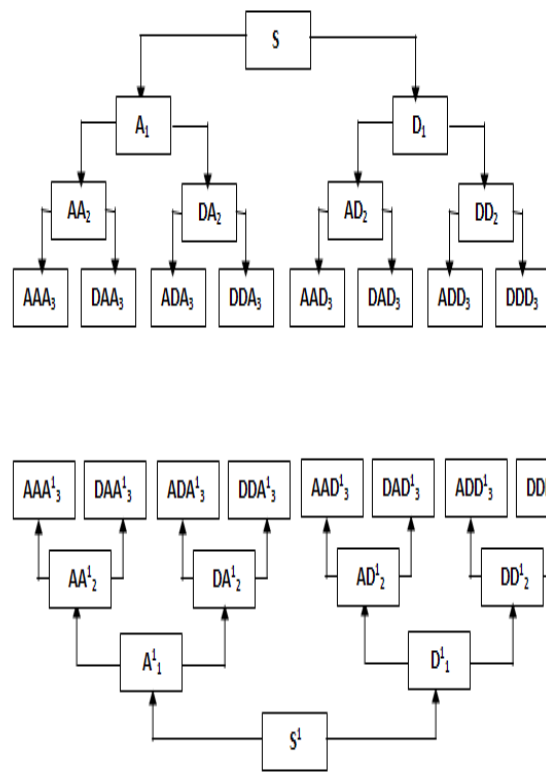

Figure 2 : Multiple DWT decomposition

Figure 3 shows the decomposition results. The barbera image is first decomposed into four sub bands of $\mathrm{LL}, \mathrm{LH}$, $\mathrm{HL}$ and HH. Further the LL sub band is decomposed into four more sub bands as shown in the figure. The LL component has the maximum information content as shown in figure 3 , the other higher order sub bands contain the edges in the vertical, horizontal and diagonal directions. An image of size $\mathrm{N} \mathrm{X} \mathrm{N}$ is decomposed to N/2 $\mathrm{X} \mathrm{N} / 2$ of four sub bands. Choosing the LL sub band and rejecting the other sub bands at the first level compresses the image by $75 \%$. Thus DWT assists in compression.
Furhter encoding increases compression ratio.

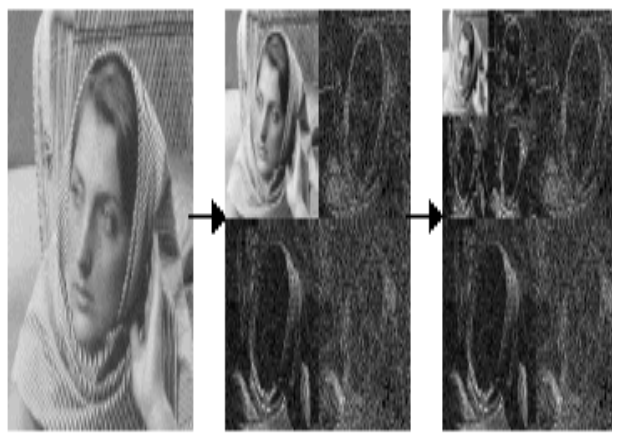

Figure 3 DWT decomposition of barbera image into hierarchical sub bands

\section{ANN with DWT for Image Compression}

Basic architecture for image compression using neural network is shown in the above figure 4 . The input image of size $64 \times 1$ is multiplied by $4 \times 64$ weight matrixes to obtain the compressed output of $4 \times 1$, at the receiver $4 \times 1$ is decompressed to $64 \times 1$ by multiplying the compressed matrix by $64 \times 4$. The table in figure 4 shows the compression ratio that can be achieved by choosing the sizes of hidden layer.

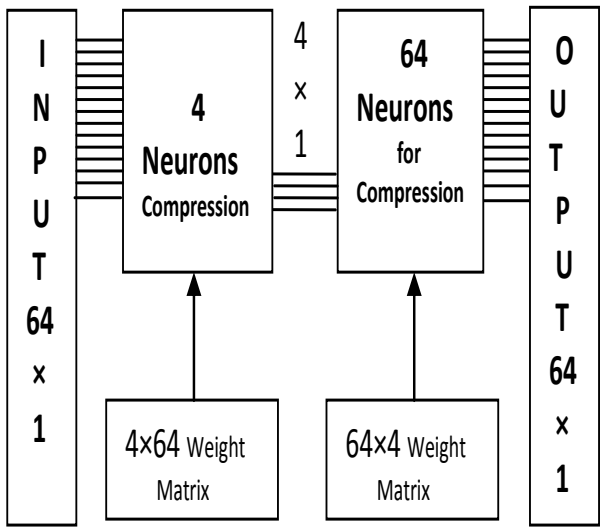

\begin{tabular}{|l|l|l|}
\hline $\begin{array}{c}\text { Network } \\
\text { size }\end{array}$ & $\begin{array}{c}\text { Size of } \\
\text { hidden layer }\end{array}$ & $\begin{array}{c}\text { Compression } \\
\text { ratio }\end{array}$ \\
\hline $64-64-64$ & 64 & $0 \%$ \\
\hline $64-32-64$ & 32 & $50 \%$ \\
\hline $64-16-64$ & 16 & $75 \%$ \\
\hline $64-08-64$ & 08 & $87.5 \%$ \\
\hline $64-04-64$ & 04 & $93.75 \%$ \\
\hline $64-01-64$ & 01 & $98.5 \%$ \\
\hline
\end{tabular}

\section{Figure 4 Neural network based image compression}

Prior to use of $\mathrm{NN}$ for compression it is required to perform training of the network, in this work back propagation training algorithm is used for obtaining the optimum weights and biases for the $\mathrm{NN}$ architecture. Based on the training, barbera image is compressed and decompressed; Figure 5 shows the input image, compressed image and decompressed image. 


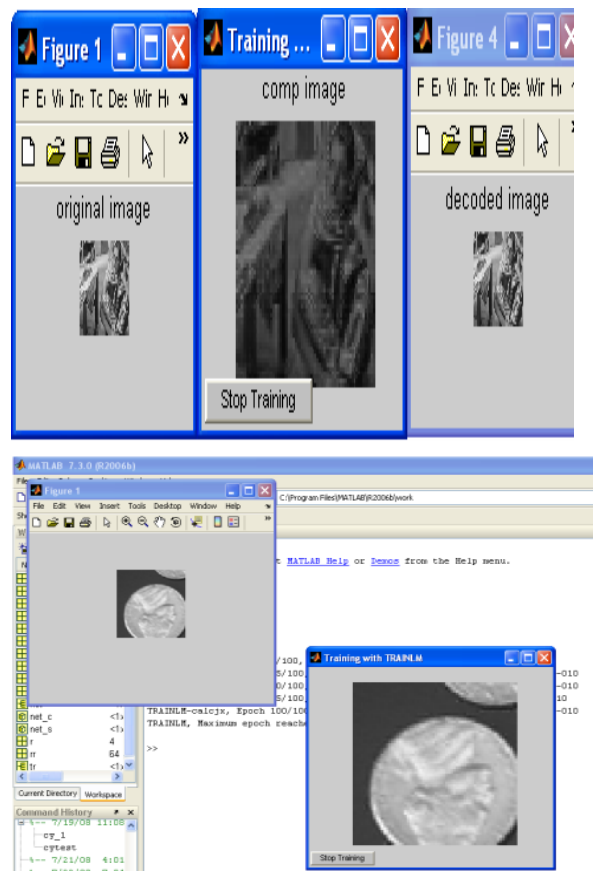

Figure 5 NN based image compression and decompression

Figure 5 also shows the input image and the decompressed image of coins image using neural network architecture. From the decompressed results shown, it is found that the checker blocks error, which exists on the decompressed image. As the input image is sub divided into $8 \times 8$ blocks and rearranged to $64 \times 1$ input matrixes, the checker block arises. This is one of the limitations of $\mathrm{NN}$ based compression. Another major limitation is the maximum compression ration which is less than $100 \%$, in order to achieve compression more than $100 \%$ and to eliminate checker box errors or blocking artifacts DWT combined with $\mathrm{NN}$ architecture is proposed for image compression.

\subsection{Proposed Technique for Image Compression}

Most of the image compression techniques use either neural networks for compression or DWT (Discrete wavelet Transform) based transformation for compression. In order to overcome the limitations of NN architecture in this work, DWT is used for image decomposition and an $\mathrm{N}$ $\mathrm{X} \mathrm{N}$ image is decomposed using DWT into hierarchical blocks the decomposition is carried out until the sub block is of size $8 \times 8$. For a image of size $64 \times 64$, first level decomposition gives rise to $32 \times 32$ (four sub bands) of sub blocks, further decomposition leads to $16 \times 16$ (sixteen sub bands), which can further decamped to $8 \times 8$ at the third hierarchy. The third level of hierarchy there are 64 sub blocks each of size $8 \times 8$. Figure 6 shows the decomposition levels of input image of size 64 x 64 .

\begin{tabular}{|l|c|l|c|l|l|l|l|}
\hline LL & LH & LL & LH & LL & LH & LL & LH \\
\hline HL & HH & HL & HH & HL & HH & HL & HH \\
\hline LL & LH & LL & LH & LL & LH & LL & LH \\
\hline HL & HH & HL & HH & HL & HH & HL & HH \\
\hline LL & LH & LL & LH & LL & LH & LL & LH \\
\hline HL & HH & HL & HH & HL & HH & HL & HH \\
\hline LL & LH & LL & LH & LL & LH & LL & LH \\
\hline HL & HH & HL & HH & HL & HH & HL & HH \\
\hline
\end{tabular}

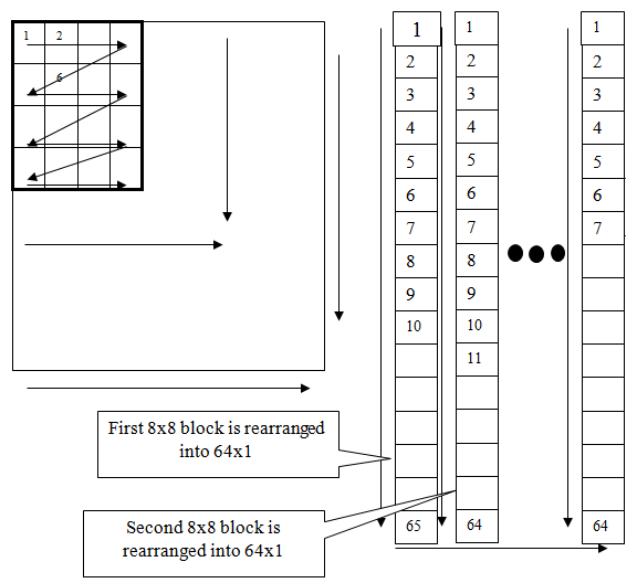

Figure 6 Decomposition of image into sub blocks using DWT

Sub blocks of $8 \times 8$ are rearranged to $64 \times 1$ block are combined together into a rearranged matrix size as shown in figure 6. The rearranged matrix is used to train the NN architecture based on back propagation algorithm. In order to train the NN architecture and to obtain optimum weights it is required to select appropriate images. The training vectors play a vital role in $\mathrm{NN}$ architecture for image compression. Figure 7 shows the training sets for NN architecture.
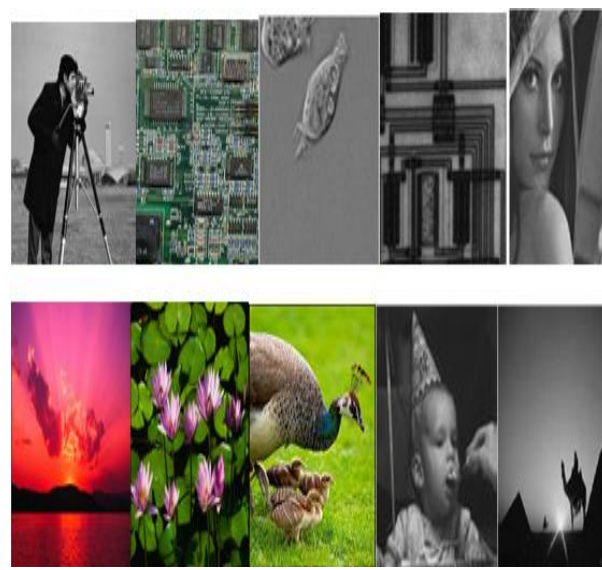

Figure 7 Training set for NN architecture

The NN architecture consisting of input layer, hidden layer and output layer. The hidden layer consists of network function of four types shown in Table 1. Similarly the 
output layer also can be any of the four network functions. It is required to choose appropriate network function.

Table 1: Neural network classification based on transfer function

\begin{tabular}{|c|c|c|}
\hline \multirow{2}{*}{$\begin{array}{l}\text { Neural } \\
\text { network } \\
\text { type }\end{array}$} & \multicolumn{2}{|c|}{ Transfer function } \\
\hline & $\begin{array}{l}\text { Hidden } \\
\text { layer }\end{array}$ & $\begin{array}{l}\text { Output } \\
\text { layer }\end{array}$ \\
\hline $\begin{array}{l}\text { Linear } \\
\text { network }\end{array}$ & Purelin & Purelin \\
\hline $\begin{array}{c}\text { Nonlinear } \\
\text { network }\end{array}$ & $\begin{array}{l}\text { Tansig or } \\
\text { logsig }\end{array}$ & $\begin{array}{l}\text { Tansig or } \\
\text { logsig }\end{array}$ \\
\hline $\begin{array}{c}\text { Hybrid } \\
\text { network }\end{array}$ & $\begin{array}{l}\text { Tansig or } \\
\text { logsig }\end{array}$ & Purelin \\
\hline
\end{tabular}

Trainrp is a network training function used in this work that updates weight and bias values according to the resilient backpropagation algorithm (Rprop). Trainlm may also be used which is also a network training function that updates weight and bias values according to Levenberg algorithm but consumes more memory.

\section{Proposed hybrid architecture}

In this work, hybrid neural network architecture which combines DWT with NN is used to image compression. The hybrid architecture is discussed in [Ramanaiah and Cyril]. The NN based compression using analog VLSI is presented in [Cyril and Pinjare]. Based on the two different papers neural network architecture is developed and is trained to compress and decompress multiple images. The DWT based image compression algorithm is combined with neural network architecture. There are several wavelet filters and neural network functions.

It is required to choose appropriate wavelets and appropriate neural network functions. In this work an experimental setup is modeled using Matlab to choose appropriate wavelet and appropriate neural network function. Based on the above parameters chosen the Hybrid Compression Algorithm is developed and is shown in Figure 8.

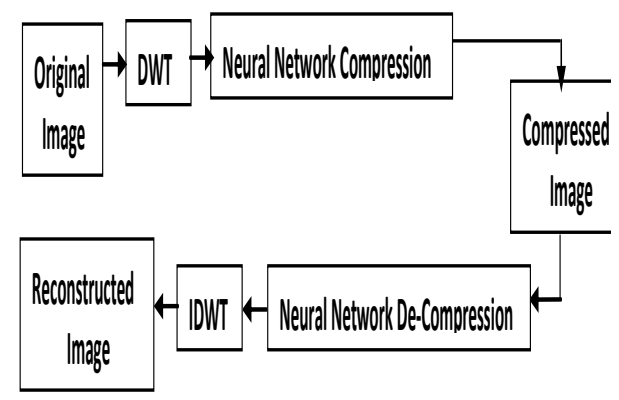

Figure 8 Proposed hybrid algorithms for image compression

Several images are considered for training the network, the input image is resized to $256 \times 256$, the resized image is transformed using DWT, 2D DWT function is used for the transformation. There is several wavelet functions, in this work Haar and dB4 wavelet functions are used. The input image is decomposed to obtain the sub band components using several stages of DWT. The DWT process is stopped until the sub band size is $8 \times 8$. The decomposed sub band components are rearranged to column vectors; the rearranged vectors are concatenated to matrix and are set at the input to the neural network. The hidden layer is realized using 4 neurons and tansig function. The weights are biases obtained after training are used to compress the input to the required size and is further processed using weights and biases in the output layer to decompress. The decompressed is further converted from vector to blocks of sub bands. The sub band components are grouped together and are transformed using inverse DWT. The transformation is done using multiple hierarchies and the original image is reconstructed. The input image and the output image is used to compute MSE, PSNR. The selection of network parameters and performances are discussed in the next section.

\section{Results and Discussion}

Training the network using the test training sets and selection of appropriate network function is carried out, Matlab model is developed and is used for analysis. Table 2 and Table 3 shows the results for Pears image for various network functions and hidden layer size.

Table 2: NN performance for various network functions for Pears

\begin{tabular}{|c|c|c|c|c|c|}
\hline Pears & \multicolumn{5}{|c|}{ MSE-Pears } \\
\hline $\begin{array}{l}\text { Netw } \\
\text { ork }\end{array}$ & $\begin{array}{l}\text { Pureli } \\
\mathrm{n} \\
- \\
\text { Pureli } \\
\mathrm{n}\end{array}$ & $\begin{array}{l}\text { Tansig } \\
- \\
\text { Tansig }\end{array}$ & $\begin{array}{l}\text { Tansi } \\
\mathrm{g} \\
- \\
\text { Pureli } \\
\mathrm{n}\end{array}$ & $\begin{array}{l}\text { Pureli } \\
\text { n } \\
- \\
\text { Tansi } \\
\text { g }\end{array}$ & $\begin{array}{l}\text { Logsi } \\
\mathrm{g} \\
- \\
\text { Logsi } \\
\mathrm{g}\end{array}$ \\
\hline [864] & $\begin{array}{l}26.77 \\
6\end{array}$ & $\begin{array}{l}6.23 \mathrm{E} \\
+02\end{array}$ & $\begin{array}{l}53.56 \\
89\end{array}$ & $\begin{array}{l}4.65 \mathrm{E} \\
+02\end{array}$ & 94 \\
\hline $\begin{array}{l}{[166} \\
4]\end{array}$ & $\begin{array}{l}16.01 \\
31 \\
\end{array}$ & $\begin{array}{l}1.76 \mathrm{E} \\
+02 \\
\end{array}$ & $\begin{array}{l}37.42 \\
3 \\
\end{array}$ & $\begin{array}{l}5.33 \mathrm{E} \\
+02\end{array}$ & $\begin{array}{l}4.53 \mathrm{E} \\
+02\end{array}$ \\
\hline $\begin{array}{l}{[326} \\
4]\end{array}$ & $\begin{array}{l}14.62 \\
3\end{array}$ & $\begin{array}{l}71.813 \\
6\end{array}$ & $\begin{array}{l}39.89 \\
7\end{array}$ & $\begin{array}{l}58.85 \\
47\end{array}$ & $\begin{array}{l}3.18 \mathrm{E} \\
+02\end{array}$ \\
\hline $\begin{array}{l}{[406} \\
4]\end{array}$ & $\begin{array}{l}15.88 \\
8\end{array}$ & $\begin{array}{l}62.185 \\
4\end{array}$ & $\begin{array}{l}40.60 \\
7\end{array}$ & $\begin{array}{l}56.29 \\
62 \\
\end{array}$ & $\begin{array}{l}1.14 \mathrm{E} \\
+02\end{array}$ \\
\hline
\end{tabular}

Table 3: NN performance for various network functions for Trees

\begin{tabular}{|c|c|c|c|c|c|}
\hline $\begin{array}{l}\mathrm{N} \\
\text { et } \\
\mathrm{W} \\
\text { or } \\
\mathrm{k}\end{array}$ & $\begin{array}{l}\text { Pure } \\
\text { lin- } \\
\text { Pure } \\
\text { lin }\end{array}$ & $\begin{array}{l}\text { Tansi } \\
\text { g- } \\
\text { Tansi } \\
\text { g }\end{array}$ & $\begin{array}{l}\text { Tansi } \\
\mathrm{g} \text { - } \\
\text { Pureli } \\
\mathrm{n}\end{array}$ & $\begin{array}{l}\text { Pure } \\
\text { lin - } \\
\text { Tans } \\
\text { ig }\end{array}$ & $\begin{array}{l}\text { Logsi } \\
\text { g- } \\
\text { Logsi } \\
\text { g }\end{array}$ \\
\hline$[8$ & 7.57 & 120.4 & $1.53 \mathrm{E}$ & 84.2 & 189.8 \\
\hline 64 & 7 & 427 & -08 & 421 & 105 \\
\hline $\begin{array}{l}{[1} \\
66\end{array}$ & $\begin{array}{l}11.1 \\
768\end{array}$ & $\begin{array}{l}58.67 \\
97\end{array}$ & $\begin{array}{l}0.741 \\
2\end{array}$ & $\begin{array}{l}74.2 \\
862\end{array}$ & $\begin{array}{l}1.51 \mathrm{E} \\
+03\end{array}$ \\
\hline 4] & & & & & \\
\hline$[3$ & 20.5 & 32.54 & 29.65 & 56.2 & 82.27 \\
\hline $\begin{array}{l}26 \\
4]\end{array}$ & 682 & 22 & 25 & 924 & 05 \\
\hline$[4$ & 11.0 & 25.67 & 44.86 & 46.4 & 70.55 \\
\hline $\begin{array}{l}06 \\
4]\end{array}$ & 607 & 74 & 34 & 044 & 17 \\
\hline
\end{tabular}

From the results presented in Table 2 and Table 3 shows that for NN architecture of [8 64] (8 neurons in hidden layer and 64 in the output layer), MSE is very less for Tansig-Purelin. Hence in this work, tansig and purelin are the two network functions for $\mathrm{NN}$ architecture that are 
adopted and hence the architecture is called as Hybrid NN architecture. Figure 9 shows the MSE, PSNR and Max Error parameters for various input block size.

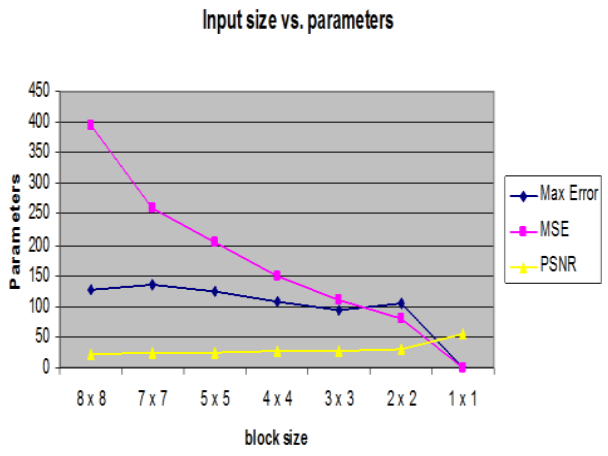

Figure 9 Input block size and NN performance

From the results shown in figure 9 it is found that lesser input layer size better is MSE, however if the input layer size less it also increases the complexity of NN architecture. Number of hierarchical levels in DWT need to be increased, hence in this work $8 \times 8$ block size is chosen, the input image is divided into $8 \times 8$ block size using DWT. Figure 10 shows the results of selection of number of hidden layers. The input layer consisting of $64 x$ 1 can be compressed to $16 \times 1$, which can be further compressed to $8 \times 1$, and further to $4 \times 1$ and can be reconstructed to $64 \times 1$ at the output layer. The results shown are analyzed for three images, from the results it is found that increasing the number of hidden layers does not improve NN compression performance. Hence the network chosen in this work consists of input layer of $64 \times 1$, hidden layer of $4 \times 1$ and output layer of $64 \times 1$. The network functions are tansig in the hidden layer and purelin in the output layer.

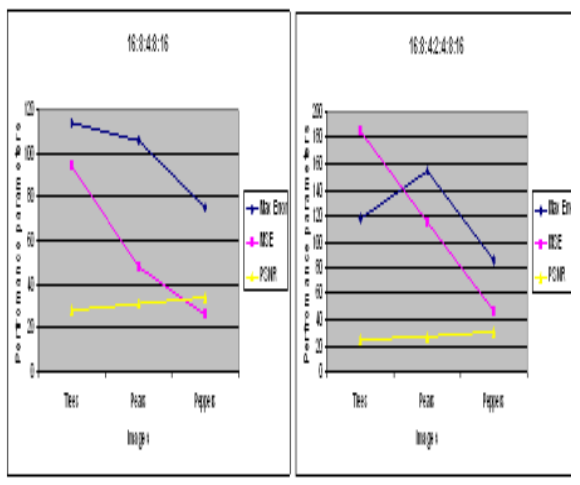

\section{Figure $10 \mathrm{NN}$ performances for various hidden layers}

In this proposed architecture, the input image is first decomposed into multiple sub blocks using hierarchical DWT architecture, the decomposed image is reordered and is processed using the $\mathrm{NN}$ architecture. The NN architecture compresses the transformed image, appropriate weights and biases are chosen for compression and decompression. Hybrid network functions are used for $\mathrm{NN}$ architecture. The decompressed image is reconstructed using IDWT. Figure 11 shows the results of image compression and decompression using the proposed hybrid architecture model. The input image is transformed into four sub bands in the first level decomposition, further is decomposed to second level of hierarchy and is shown in figure 11. The decomposed image is rearranged into column matrix, and is shown in figure 11. The compressed data using $\mathrm{NN}$ architecture is decompressed using output layer. The output obtained is further rearranged to sub blocks and is inverse transformed using inverse DWT. The output obtained is shown in figure, along with the reconstructed image.
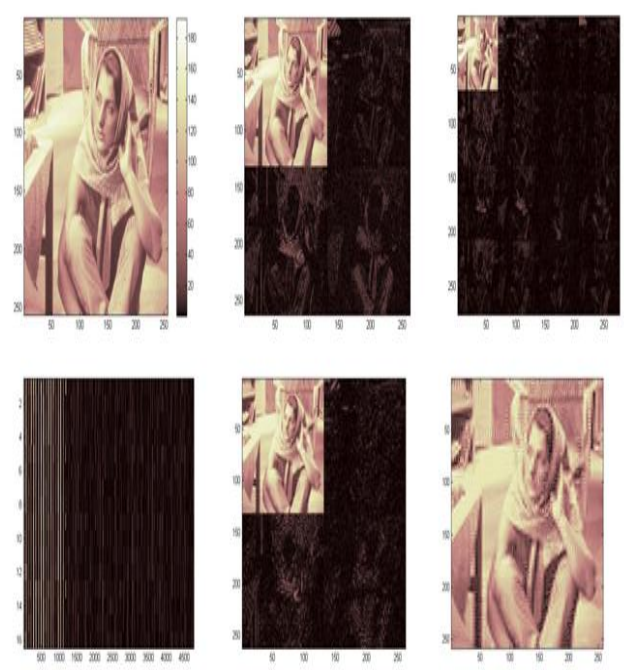

Figure 11: Results of hybrid neural network architecture
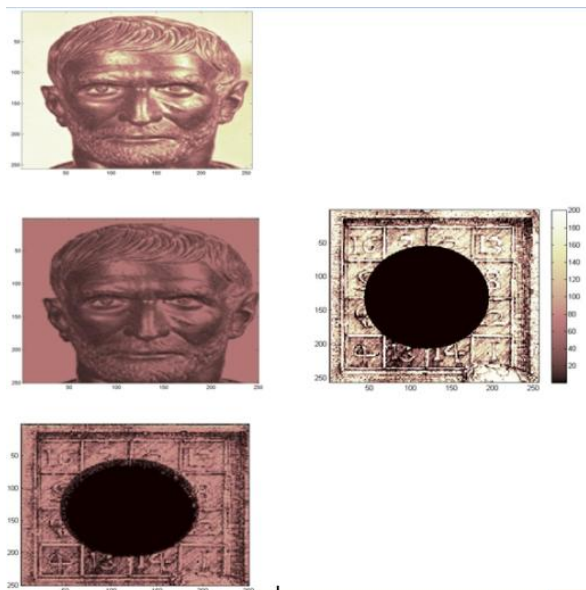

Figure 12 Input image and reconstructed image 
Table 4:

\begin{tabular}{|l|l|l|l|l|}
\hline $\begin{array}{l}\text { Sl } \\
\text { no. }\end{array}$ & $\begin{array}{l}\text { Test } \\
\text { Image }\end{array}$ & $\begin{array}{l}\text { Image } \\
\text { MSE } \\
\text { Nith } \\
\text { only) }\end{array}$ & $\begin{array}{l}\text { Refer } \\
\text { ence }\end{array}$ & $\begin{array}{l}\text { Image } \\
\text { MSE } \\
\text { Nith } \\
\text { NN and } \\
\text { DWT) }\end{array}$ \\
\hline 1 & cameraman & 321 & 301 & 262 \\
\hline 2 & board & 1590 & 1289 & 958 \\
\hline 3 & cell & 39 & 34 & 26 \\
\hline 4 & circuit & 24 & 21 & 14 \\
\hline 5 & Lena & 201 & 190 & 100 \\
\hline 6 & sun & 278 & 149 & 115 \\
\hline 7 & girl & 67 & 51 & 42 \\
\hline 8 & Blue hills & 38 & 31 & 22 \\
\hline 9 & Sunset & 51 & 47 & 39 \\
\hline 10 & Water lilies & 56 & 42 & 31 \\
\hline 11 & Winter & 89 & 52 & 47 \\
\hline 12 & Drawing & 260 & 232 & 170 \\
\hline 13 & College & 180 & 126 & 97 \\
\hline 14 & Garden & 163 & 145 & 87 \\
\hline 15 & My photo & 320 & 234 & 197 \\
\hline 16 & Holi & 289 & 256 & 175 \\
\hline 17 & Bhagavadgetha & 98 & 78 & 65 \\
\hline 18 & Devinecouple & 143 & 101 & 80 \\
\hline 19 & krishna & 29 & 19 & 7 \\
\hline 20 & Goddess & 76 & 53 & 45 \\
\hline
\end{tabular}

Table 4 summarizes the MSE results for various test images using the hybrid architecture.

The results compare the performances of NN architecture, reference design and the present work. With the choice of appropriate wavelet filters (Haar, db4), choice of decomposition levels, number of hidden layers and network function the proposed architecture is superior compared with all the other architectures.

From the results presented in table for all the 20 images considered proposed network achieves less MSE compared with the reference design. The input image is decomposed using DWT and is compressed using NN architecture, this introduces delay and hence high speed architectures are required to implement for real time applications.

\section{Conclusion}

Use of $\mathrm{NN}$ for image compression has superior advantage compared with classical techniques, however the NN architecture requires image to be decomposed to several blocks of each $8 \times 8$, and hence introduces blocking artifact errors and checker box errors in the reconstructed image. In order to overcome the checker errors in this work, DWT for image decomposition prior to image compression using $\mathrm{NN}$ architecture is used. In this work, hybrid architecture that combines NN with DWT is proposed and the input image is used to train the network. The network architecture is used to compress and decompress several image and it is proven to achieve better MSE compared with reference design. The hybrid technique uses hidden layer consisting of tansig function and output layer with purelin function to achieve better MSE. The proposed architecture is suitable for real time application of image compression and decompression.

\section{ACKNOWLEDGMENTS}

The authors would like to acknowledge Dr. Cyril Prasanna Raj P. for his valuable support during this work.

\section{REFERENCES}

[1] Q. Zang, Wavelet Network in Nonparametric Estimation. IEEE Trans. Neural Networks, 8(2):227- 236, 1997

[2] Q. Zang and A. Benveniste, Wavelet networks. IEEE Trans. Neural Networks, vol. 3, pp. 889-898, 1992.

[3] A. Grossmann and B. Torrésani, Les ondelettes, Encyclopedia Universalis, 1998.

[4] R. Ben Abdennour, M. Ltaïef and M. Ksouri. uncoefficient d'apprentissage flou pour les réseaux deneurones artificiels, Journal Européen des Systèmes Automatisés, Janvier 2002.

[5] M. Chtourou. Les réseaux de neurones, Support de cours DEA A-II, Année Universitaire 2002/2003.

[6] Y. Oussar. Réseaux d'ondelettes et réseaux de neurones pour la modélisation statique et dynamique de processus, Thèse de doctorat, Université Pierre et Marie Curie, juillet 1998.

[7] R. Baron. Contribution à l'étude des réseaux d'ondelettes, Thèse de doctorat, Ecole Normale Supérieure de Lyon, Février 1997.

[8] C. Foucher and G. Vaucher. Compression d'images et réseaux de neurones, revue Valgo $n^{\circ} 01-02,17-19$ octobre 2001,Ardèche.

[9] J. Jiang. Image compressing with neural networks - A survey, Signal processing Image communication, ELSEVIER, vol.14, n9, 1999, p. 737-760.

[10] S. Kulkarni, B. Verma and M. Blumenstein. Image Compression Using a Direct Solution Method Based NeuralNetwork, The Tenth Australian Joint Conference on Artificial Intelligence,Perth, Australia, 1997, p. 114-119. 
[11] G. Lekutai. Adaptive Self-tuning Neuro Wavelet Network Controllers, Thèse de Doctorat, BlacksburgVirgina, Mars 1997.

[12] R.D. Dony and S. Haykin. Neural network approaches to imagcompression, Proceedings of the IEEE, V83, N², Février, 1995, p. 288-303.

[13] A. D'souza Winston and Tim Spracklen. Application of Artificial Neural Networks for real time Compression, 8th International Conference On Neural Processing, Shanghai, Chine, 14-18 Novembre 2001.

[14] Ch. Bernard, S. Mallat and J-J Slotine. Wavelet Interpolation NetworksInternational Workshop on CAGD and wavelet methods for ReconstructingFunctions, Montecatini, 15-17 Juin 1998.

[15] D. Charalampidis. Novel Adaptive Image Compression, Workshop on Information and Systems Technology, Room 101, TRAC Building, University of New Orleans, 16 Mai 2003.

[16] M. J. Nadenau, J. Reichel, and M. Kunt, "Wavelet Based Color Image CompressioExploiting the Contrast Sensitivity Function", IEEE Transactions Image Processing, vol. 12, no.1, 2003, pp. 58-70.

[17] K. Ratakonda and N. Ahuja, "Lossless Image Compression with Multiscale Segmentation", IEEE Transactions Image Processing, vol. 11, no.11, 2002,pp. 1228-1237.

[18] K. H. Talukder and K. Harada, "Haar Wavelet Based Approach for ImageCompression and Quality Assessment of Compressed Image", IAENG International Journal of Applied Mathematics, 2007.

[19] Bo-Luen Lai and Long-Wen Chang, “Adaptive Data Hiding for Images Based on Haar Discrete Wavelet Transform", Lecture Notes in Computer Science, Springer-Verlag, vol. 4319,2006, pp. 1085-1093.

[20] S. Minasyan, J. Astola and D. Guevorkian, "An Image CompressionScheme Based on Parametric Haar-like Transform", ISCAS 2005. IEEE International Symposium on Circuits and Systems, 2005, pp. 2088-2091.

[21] Z. Ye, H. Mohamadian and Y.Ye, "Information Measures for Biometric Identification via 2D Discrete WaveletTransform", Proceedings of the 3rd Annual IEEE Conference on Automation Science and Engineering, CASE’2007, 2007, pp. 835-840.

[22] S. Osowski， R. Waszczuk，P. Bojarczak, "Image compression using feed forward neural networks Hierarchical approach" Lecture Notes in Computer Science, Book Chapter, Springer- Verlag, vol. 3497, 2006, pp. 1009- 1015.

[23] M. Liying and K. Khashayar, "Adaptive Constructive Neural Networks Using Hermite Polynomials for Image Compression", Lecture Notes in Computer Science, Springer-Verlag, vol. 3497, 2005, pp. 713722.
[24] R. Cierniak, "Image Compression Algorithm Based on Soft Computing Techniques", Lecture Notes in Computer Science, Springer-Verlag, vol. 3019,2004, pp. 609-617.

[25] B. Northan, and R.D. Dony, "Image Compression with a multiresolution neural network", Canadian Journal of Electrical and Computer Engineering, Vol. 31, No. 1, 2006, pp. 49-58.

[26] S. Veisi and M. Jamzad, "Image Compression with Neural Networks Using Complexity Level of Images", Proceedings of the $5^{\text {th }}$ International Symposium on image and Signal Processing and Analysis, ISPA07, IEEE, 2007, pp. 282-287.

[27] I. Vilovic, "An Experience in Image Compression Using Neural Networks", $48^{\text {th }}$ International Symposium ELMAR 2006 focused on Multimedia Signal Processing and Communications, IEEE, 2006, pp. 95-98.

[28] J. Mi, D. Huang, "Image Compression using Principal Component Neural Network", $8^{\text {th }}$ International Conference on Control, Automation, Robotics and Vision, IEEE, 2004,pp. 698-701.

[29] R. Ashraf and M. Akbar, "Absolutely lossless compression of medical images", 27th Annual Conference Proceedings of the 2005 IEEE Engineering in Medicine and Biology, IEEE, 2005, pp. 4006-4009.

[30] A. Khashman and K. Dimililer, "Neural Networks Arbitration for Optimum DCT Image Compression", Proceeding of the IEEE International Conference on 'Computer as a Tool' EUROCON'07, 2007, pp. 151156.

[31] A. Khashman and K. Dimililer, "Intelligent System for Image Compression", Proceeding of 9th International Conference on Enterprise Information Systems, ICEIS 2007, 2007,pp. 451-454.

[32] A. Khashman and K. Dimililer, "Comparison Criteria for Optimum Image Compression", Proceeding of the IEEE International Conference on "Computer as a Tool’ EUROCON'05, vol. 2, 2005, pp. 935-938.

[33] A. Khashman and K. Dimililer, "Haar Image Compression Using a Neural Network", Proceedings of the WSEAS Int. Applied Computing Conference (ACC'08), Istanbul, Turkey, 27-29 May 2008.

[34] A. Khashman, B. Sekeroglu, and K Dimililer, "Intelligent Identification System for Deformed Banknotes", WSEAS Transactions on Signal Processing, ISSN 1790-5022, Issue 3, Vol. 1, 2005.

[35] K.Venkata Ramanaiah and Cyril Prasanna Raj, "ASIC Implementation of Neural Network Based Image Compression," International Journal of Computer Theory and Engineering vol. 3, no. 4, pp. 494-498, 2011 